\title{
Après 2015 : tout reste à construire, enfin !
}

L'année 2015 démontre un niveau inédit d'engagement des gouvernements du monde en faveur du développement durable, tant avec les 17 objectifs de développement durable validés en septembre ${ }^{1}$ qu'avec l'accord trouvé à Paris en décembre à la COP $21^{2}$. Si ces accords sont insuffisants, ce sont les meilleurs possibles, reflétant ce que les États de la planète, dans la diversité de leurs projets politiques et malgré leurs conflits économiques, politiques ou culturels, ont été capables de produire. Il était inespéré que ces gouvernements acceptent de se donner un agenda de transformation à 15 ans, portant non plus sur les 8 objectifs du Millénaire pour le développement - accord trouvé en 2000 a minima sur les besoins et les services les plus essentiels pour les pays du Sud-, mais sur un ensemble d'objectifs universels et beaucoup plus normatifs pour les modèles de développement, qu'il s'agisse des systèmes agricoles et alimentaires durables ou de la réduction des inégalités.

L'accord trouvé à Paris à l'issue de la COP 21 a également un sens politique très fort: il constitue une véritable refondation de l'action internationale pour le climat.

Premier trait politique remarquable, les pays du Sud, émergents comme moins avancés, ont accepté de s'engager en matière de réduction des émissions de gaz à effet de serre, franchissant ainsi un pas majeur par rapport à la situation antérieure où seuls les pays du Nord étaient tenus d'agir. La nouvelle interprétation du principe de responsabilités communes mais différenciées est qu'il est urgent que tous agissent, à la mesure de leurs moyens et en fonction de leurs responsabilités passées. L'ampleur du défi est tel, tant en matière d'atténuation que d'adaptation, que tous les pays doivent envisager des trajectoires de transformation radicale de leurs économies et de leurs sociétés.

L'autre fait politique marquant est qu'on a enfin cessé de croire qu'une conférence des Nations unies pouvait imposer des changements majeurs dans les politiques

\footnotetext{
1 http://www.un.org/sustainabledevelopment/fr/objectifsde-developpement-durable.

2 http://www.cop21.gouv.fr.
}

nationales. Impossible qu'une telle conférence permette de partager le budget carbone mondial en imposant à l'Inde ou à tout autre pays un changement radical de trajectoire économique, orthogonal aux arbitrages politiques nationaux. Impossible aussi de décréter un prix mondial du carbone : derrière l'idée simple du signal prix, il faudrait bâtir une institution particulièrement complexe, le marché de permis d'émissions, et surtout, il faudrait négocier l'allocation initiale de ces droits entre pays ou entre secteurs, ce qui revient à une négociation politiquement impossible. Ce qui s'est reconstruit depuis Copenhague en 2009, c'est une perspective de coopération internationale complètement inversée, où les États déclarent dans leur contribution nationale (INDC en anglais ${ }^{3}$ ), leurs engagements de lutte contre le changement climatique, reflet des arbitrages politiques propres à chaque pays. La coopération internationale et les transferts financiers Nord-Sud peuvent alors être repensés totalement comme un levier d'accélération pour la transformation de nos économies et de nos sociétés.

Pour cela, il fallait un accord pour créer la confiance entre tous les États : d'où l'importance d'un mécanisme de crédibilisation des engagements, qui, même insuffisants, doivent au moins être tenus. Le système de transparence mis en place est soucieux de préserver la souveraineté des pays, mais suffisamment crédible pour qu'aucun acteur national ne puisse plus réduire l'ambition des politiques climatiques de sa nation en utilisant l'argument que d'autres États, particulièrement les grands émetteurs, ne feraient rien.

Un autre pilier majeur de cet accord est qu'il consacre la nécessité de placer l'adaptation au même niveau $\mathrm{d}$ 'ambition que les réductions d'émissions. C'est l'indispensable contrepartie, pour les pays du Sud et, en particulier les plus vulnérables, au fait que les engagements soient devenus universels. C'est aussi l'indispensable reconnaissance que même avec $2{ }^{\circ} \mathrm{C}$ d'augmentation de la température moyenne mondiale, les conséquences des changements climatiques pourront être très graves pour

3 Intended Nationally Determined Contribution. 
de nombreux territoires. C'est surtout la possibilité donnée, au-delà des transferts financiers essentiels pour ces pays, à une véritable coopération internationale pour appuyer les politiques d'adaptation à toutes les échelles, et à mettre en place des stratégies innovantes combinant adaptation et atténuation, pour des systèmes urbains ou des territoires ruraux, révélant un immense potentiel pour renouveler profondément les modèles de développement (voir, par exemple, la place donnée à l'agrobiodiversité pour l'adaptation et l'atténuation dans l'INDC mexicaine).

La capacité à innover et à augmenter l'ambition des politiques nationales constitue le ressort essentiel de l'accord de Paris. Le cumul des émissions en 2030, envisagées en 2015 par les États de la planète, rend impossible la trajectoire qui réduirait ensuite drastiquement les émissions globales entre 2030 et 2050 pour rester sous les $2{ }^{\circ} \mathrm{C}$, sans même parler des $1,5^{\circ} \mathrm{C}$ indispensables pour la survie de certains pays et de certains écosystèmes. Pour que le régime de gouvernance établi à Paris en 2015 fonctionne, il a aussi fallu mettre en place un mécanisme de revue régulière à la hausse des engagements des pays pour augmenter l'ambition suffisamment vite et faire converger les anticipations des différents agents économiques en faveur d'une sortie de l'économie intensive en carbone.

Les premiers signaux donnés en 2015 sont encourageants, qu'il s'agisse des tendances boursières relatives des énergies renouvelables et des énergies fossiles, ou des institutions financières qui commencent à considérer les investissements liés aux énergies fossiles comme sans avenir. Nous sommes engagés dans une course contre la montre, comme le soulignait l'éditorial du numéro spécial de NSS de juin 2015, consacré aux enjeux de la COP $21^{4}$.

Alors, 2015 est-elle l'année d'un basculement? Il paraît urgent de miser sur l'élan donné par la COP 21, pour reposer avec force les questions de développement durable comme remise en cause des trajectoires de développement de nos sociétés, et donner ainsi une légitimité aux acteurs porteurs de trajectoires innovantes, alternatives, pour leur ville, leur secteur, leur territoire, leur pays, leur région. Certes, utiliser la COP 21 comme une opportunité pour requestionner le projet politique de nos sociétés ne doit pas nous faire oublier le risque de ne tout lire qu'à travers le filtre du carbone, alors que les émissions de gaz à effet de serre ne sont qu'un indicateur de dysfonctionnement parmi d'autres. En particulier dans les agendas scientifiques, s'il est indispensable de se mobiliser pour aider à accroître l'ambition des politiques climatiques, il serait terrible qu'elles drainent tous les moyens de la recherche et assèchent les autres formes de questionnement sur nos sociétés et leur environnement.

2015 a aussi révélé l'importance de la mobilisation sur la problématique du climat de toutes les communautés scientifiques, y compris et en particulier les sciences sociales dans toute leur diversité, comme en a témoigné le numéro spécial de NSS de juin 2015 : changements de comportements, mobilisations collectives, actions organisées à diverses échelles, réflexion politique et critique, nouvelles formulations des problèmes publics, nouvelles solutions, évaluations des performances et des effets induits de ces innovations... C'est tout cela que doit recouvrir « l'agenda des solutions » qui pourra faire fonctionner l'accord de Paris. Est ouvert maintenant un énorme chantier de contributions interdisciplinaires pour aider à construire les arrangements politiques locaux, différenciés autant que les INDCs sont euxmêmes des expressions diversifiées, liées aux histoires sociopolitiques des pays et à leurs enjeux spécifiques. Toutes les sciences seront nécessaires pour concilier cette indispensable différenciation des enjeux, la prise en compte de la plus petite des voix, porteuse d'une innovation ou d'une vulnérabilité particulière, et la nécessité d'une transformation globale et rapide pour reboucler l'équation au niveau global et rester ainsi dans la trajectoire des $2^{\circ} \mathrm{C}$.

Sébastien Treyer

\footnotetext{
4 Aubertin, C., Damian, M., Magny, M., Millier, C., Theys, J., Treyer, S. (Eds), 2015. Les enjeux de la conférence de Paris. Penser autrement la question climatique, Natures Sciences Sociétés, vol. 23, suppl, http:/ /www.nss-journal.org/fr/articles/nss/abs/2015/ $02 /$ contents/contents.html.
} 